\title{
Research Article \\ On the Existence Result for System of Generalized Strong Vector Quasiequilibrium Problems
}

\author{
Somyot Plubtieng and Kanokwan Sitthithakerngkiet \\ Department of Mathematics, Faculty of Science, Naresuan University, Phitsanulok 65000, Thailand \\ Correspondence should be addressed to Somyot Plubtieng, somyotp@nu.ac.th
}

Received 3 December 2010; Accepted 12 January 2011

Academic Editor: Qamrul Hasan Ansari

Copyright (C) 2011 S. Plubtieng and K. Sitthithakerngkiet. This is an open access article distributed under the Creative Commons Attribution License, which permits unrestricted use, distribution, and reproduction in any medium, provided the original work is properly cited.

We introduce a new type of the system of generalized strong vector quasiequilibrium problems with set-valued mappings in real locally convex Hausdorff topological vector spaces. We establish an existence theorem by using Kakutani-Fan-Glicksberg fixed-point theorem and discuss the closedness of strong solution set for the system of generalized strong vector quasiequilibrium problem. The results presented in the paper improve and extend the main results of Long et al. (2008).

\section{Introduction}

The equilibrium problem is a generalization of classical variational inequalities. This problem contains many important problems as special cases, for instance, optimization, Nash equilibrium, complementarity, and fixed-point problems (see [1-3] and the references therein). Recently, there has been an increasing interest in the study of vector equilibrium problems. Many results on existence of solutions for vector variational inequalities and vector equilibrium problems have been established (see, e.g., [4-16]).

Let $X$ and $Z$ be real locally convex Hausdorff space, $K \subset X$ a nonempty subset and $C \subset Z$ be a closed convex pointed cone. Let $F: K \times K \rightarrow 2^{Z}$ be a given set-valued mapping. Ansari et al. [17] introduced the following set-valued vector equilibrium problems (VEPs) to find $x \in K$ such that

$$
F(x, y) \nsubseteq-\operatorname{int} C \quad \forall y \in K,
$$


or to find $x \in K$ such that

$$
F(x, y) \subset C \quad \forall y \in K
$$

If int $C$ is nonempty, and $x$ satisfies (1.1), then we call $x$ a weak efficient solution for (VEP), and if $x$ satisfies (1.2), then we call $x$ a strong solution for VEP. Moreover, they also proved an existence theorem for a strong vector equilibrium problem (1.2) (see [17]).

In 2000, Ansari et al. [5] introduced the system of vector equilibrium problems (SVEPs), that is, a family of equilibrium problems for vector-valued bifunctions defined on a product set, with applications in vector optimization problems and Nash equilibrium problem [11] for vector-valued functions. The (SVEP) contains system of equilibrium problems, systems of vector variational inequalities, system of vector variational-like inequalities, system of optimization problems and the Nash equilibrium problem for vectorvalued functions as special cases. But, by using (SVEP), we cannot establish the existence of a solution to the Debreu type equilibrium problem [7] for vector-valued functions which extends the classical concept of Nash equilibrium problem for a noncooperative game. Moreover, Ansari et al. [18] introduced the following concept of system of vector quasiequilibrium problems.

Let $I$ be any index set and for each $i \in I$, let $X_{i}$ be a topological vector space. Consider a family of nonempty convex subsets $\left\{K_{i}\right\}_{i \in I}$ with $K_{i} \subset X_{i}$. We denote by $K=\prod_{i \in I} K_{i}$ and $X=\prod_{i \in I} X_{i}$. For each $i \in I$, let $Y_{i}$ be a topological vector space and let $C_{i}: K \rightarrow 2^{Y_{i}}$ and $S_{i}: K \rightarrow 2^{K_{i}}$ be multivalued mappings and $F_{i}: K \times K \rightarrow Y_{i}$ be a bifunction. The system of vector quasiequilibrium problems (SVQEPs), that is, to find $x \in K$ such that for each $i \in I$,

$$
x_{i} \in S_{i}(x): F_{i}\left(x, y_{i}\right) \notin-\operatorname{int} C_{i}(x) \quad \forall y_{i} \in S_{i}(x)
$$

If $S_{i}(x)=K_{i}$ for all $x \in K$, then (SVQEP) reduces to (SVEP) (see [5]) and if the index set $I$ is singleton, then (SVQEP) becomes the vector quasiequilibrium problem. Many authors studied the existence of solutions for systems of (vector) quasiequilibrium problems, see, for example, [19-23] and references therein.

On the other hand, it is well known that a strong solution of vector equilibrium problem is an ideal solution, It is better than other solutions such as efficient solution, weak efficient solution, proper efficient solution and supper efficient solution (see [13]). Thus, it is important to study the existence of strong solution and properties of the strong solution set. In general, the ideal solutions do not exist.

Very recently, the generalized strong vector quasiequilibrium problem (GSVQEPs) is introduced by Long et al. [16]. Let $X, Y$, and $Z$ are real locally convex Hausdorff topological vector spaces, $K \subset X$ and $D \subset Y$ are nonempty compact convex subsets, and $C \subset Z$ is a nonempty closed convex cone. Let $S: K \rightarrow 2^{K}, T: K \rightarrow 2^{D}$, and $F: K \times D \times K \rightarrow 2^{Z}$ are three set-valued mappings. They considered the GSVQEP: finding $x \in K, y \in T(x)$ such that $x \in S(x)$ and

$$
F(x, y, z) \subset C, \quad \forall z \in S(x)
$$

Moreover, they gave an existence theorem for a generalized strong vector quasiequilibrium problem without assuming that the dual of the ordering cone has a weak ${ }^{*}$ compact base. 
Motivated and inspired by research works mentioned above, in this paper, we introduce a different kind of systems of generalized strong vector quasiequilibrium problem without assuming that the dual of the ordering cone has a weak ${ }^{*}$ compact base. Let $X, Y$, and $Z$ are real locally convex Hausdorff topological vector spaces, $K \subset X$ and $D \subset Y$ are nonempty compact convex subsets, and $C \subset Z$ is a nonempty closed convex cone. We also suppose that $S_{1}, S_{2}: K \rightarrow 2^{K}, T_{1}, T_{2}: K \rightarrow 2^{D}$ and $F_{1}, F_{2}: K \times D \times K \rightarrow 2^{Z}$ are set-valued mappings. We consider the following system of generalized strong vector quasiequilibrium problem (SGSVQEPs): finding $(\bar{x}, \bar{u}) \in K \times K$ and $\bar{v} \in T_{1}(\bar{x}), \bar{y} \in T_{2}(\bar{u})$ such that $\bar{x} \in S_{1}(\bar{x})$, $\bar{u} \in S_{2}(\bar{u})$ satisfying

$$
\begin{aligned}
& F_{1}(\bar{x}, \bar{y}, z) \subset C \quad \forall z \in S_{1}(\bar{x}), \\
& F_{2}(\bar{u}, \bar{v}, z) \subset C \quad \forall z \in S_{2}(\bar{u}) .
\end{aligned}
$$

We call this $(\bar{x}, \bar{u})$ a strong solution for the (SGSVQEP).

At a quick glance, our required solution seems to be similar to such a thing of Ansari et al. $[5,18]$, in the case of $I=\{1,2\}$ and $K_{1}=K_{2}$. In fact, however, the main different point comes from the independent choice of coordinate. In this paper, we establish an existence theorem of strong solution set for the system of generalized strong vector quasiequilibrium problem by using Kakutani-Fan-Glicksberg fixed-point theorem and discuss the closedness of the solution set. Moreover, we apply our result to obtain the result of Long et al. [16].

\section{Preliminaries}

Throughout this paper,we suppose that $X, Y$, and $Z$ are real locally convex Hausdorff topological vector spaces, $K \subset X$ and $D \subset Y$ are nonempty compact convex subsets, and $C \subset Z$ is a nonempty closed convex cone. We also suppose that $S_{1}, S_{2}: K \rightarrow 2^{K}$, $T_{1}, T_{2}: K \rightarrow 2^{D}$, and $F_{1}, F_{2}: K \times D \times K \rightarrow 2^{Z}$ are set-valued mappings.

Definition 2.1. Let $X$ and $Y$ be two topological vector spaces and $K$ a nonempty subset of $X$ and let $F: K \rightarrow 2^{Y}$ be a set-valued mapping.

(i) $F$ is called upper $C$-continuous at $x_{0} \in K$ if, for any neighbourhood $U$ of the origin in $Y$, there is a neighbourhood $V$ of $x_{0}$ such that, for all $x \in V$,

$$
F(x) \subset F\left(x_{0}\right)+U+C .
$$

(ii) $F$ is called lower $C$-continuous at $x_{0} \in K$ if, for any neighbourhood $U$ of the origin in $Y$, there is a neighbourhood $V$ of $x_{0}$ such that, for all $x \in V$,

$$
F\left(x_{0}\right) \subset F(x)+U-C .
$$

Definition 2.2. Let $X$ and $Y$ be two topological vector spaces and $K$ a nonempty convex subset of $X$. A set-valued mapping $F: K \rightarrow 2^{Y}$ is said to be properly $C$-quasiconvex if, for any $x, y \in K$ and $t \in[0,1]$, we have

$$
\text { either } F(x) \subset F(t x+(1-t) y)+C \quad \text { or } \quad F(y) \subset F(t x+(1-t) y)+C
$$


Definition 2.3. Let $X$ and $Y$ be two topological vector spaces, and $T: X \rightarrow 2^{Y}$ be a set-valued mapping.

(i) $T$ is said to be upper semicontinuous at $x \in X$ if, for any open set $V$ containing $T(x)$, there exists an open set $U$ containing $x$ such that, for all $t \in U, T(t) \subset V$; $T$ is said to be upper semicontinuous on $X$ if it is upper semicontinuous at all $x \in X$.

(ii) $T$ is said to be lower semicontinuous at $x \in X$ if, for any open set $V$ with $T(x) \cap V \neq \emptyset$, there exists an open set $U$ containing $x$ such that, for all $t \in U, T(t) \cap V \neq \emptyset ; T$ is said to be lower semicontinuous on $\mathrm{X}$ if it is lower semicontinuous at all $x \in \mathrm{X}$.

(iii) $T$ is said to be continuous on $X$ if, it is at the same time upper semicontinuous and lower semicontinuous on $X$.

(iv) $T$ is said to be closed if the graph, $\operatorname{Graph}(T)$, of $T$, that is, $\operatorname{Graph}(T)=\{(x, y): x \in X$ and $y \in T(x)\}$, is a closed set in $X \times Y$.

Lemma 2.4 (see [12]). Let $K$ be a nonempty compact subset of locally convex Hausdorff vector topology space E. If $S: K \rightarrow 2^{K}$ is upper semicontinuous and for any $x \in K, S(x)$ is nonempty, convex and closed, then there exists an $x^{*} \in K$ such that $x^{*} \in S\left(x^{*}\right)$.

Lemma 2.5 (see [24]). Let $X$ and $Y$ be two Hausdorff topological vector spaces and $T: X \rightarrow 2^{Y}$ be a set-valued mapping. Then, the following properties hold:

(i) if $T$ is closed and $\overline{T(X)}$ is compact, then $T$ is upper semicontinuous, where $T(X)=$ $\bigcup_{x \in X} T(x)$ and $\bar{E}$ denotes the closure of the set $E$,

(ii) if $T$ is upper semicontinuous and for any $x \in X, T(x)$ is closed, then $T$ is closed,

(iii) $T$ is lower semicontinuous at $x \in X$ if and only iffor any $y \in T(x)$ and any net $\left\{x_{\alpha}\right\}, x_{\alpha} \rightarrow$ $x$, there exists a net $\left\{y_{\alpha}\right\}$ such that $y_{\alpha} \in T\left(x_{\alpha}\right)$ and $y_{\alpha} \rightarrow y$.

\section{Main Results}

In this section, we apply Kakutani-Fan-Glicksberg fixed-point theorem to prove an existence theorem of strong solutions for the system of generalized strong vector quasiequilibrium problem. Moreover, we also prove the closedness of strong solution set for the system of generalized strong vector quasiequilibrium problem.

Theorem 3.1. For each $i=\{1,2\}$, let $S_{i}: K \rightarrow 2^{K}$ be continuous set-valued mappings such that for any $x \in K, S_{i}(x)$ are nonempty closed convex subsets of $K$. Let $T_{i}: K \rightarrow 2^{D}$ be upper semi continuous set-valued mappings such that for any $x \in K, T_{i}(x)$ are nonempty closed convex subsets of $D$ and $F_{i}: K \times D \times K \rightarrow 2^{Z}$ be set-valued mappings satisfy the following conditions:

(i) for all $(x, y) \in K \times D, F_{i}\left(x, y, S_{i}(x)\right) \subset C$,

(ii) for all $(y, z) \in D \times K, F_{i}(\cdot, y, z)$ are properly $C$-quasiconvex,

(iii) $F_{i}(\cdot, \cdot, \cdot)$ are upper $C$-continuous,

(iv) for all $y \in D, F_{i}(\cdot, y, \cdot)$ are lower $(-C)$-continuous.

Then, SGSVQEP has a solution. Moreover, the set of all strong solutions is closed. 
Proof. For any $(x, y) \in K \times D$, define set-valued mappings $A, B: K \times D \rightarrow 2^{K}$ by

$$
\begin{aligned}
& A(x, y)=\left\{a \in S_{1}(x): F_{1}(a, y, z) \subset C, \forall z \in S_{1}(x)\right\}, \\
& B(x, y)=\left\{b \in S_{2}(x): F_{2}(b, y, z) \subset C, \forall z \in S_{2}(x)\right\} .
\end{aligned}
$$

Step 1. Show that $A(x, y)$ and $B(x, y)$ are nonempty.

For any $x \in K$, we note that $S_{1}(x)$ and $S_{2}(x)$ are nonempty. Thus, for any $(x, y) \in K \times D$, we have $A(x, y)$ and $B(x, y)$ are nonempty.

Step 2. Show that $A(x, y)$ and $B(x, y)$ are convex subsets of $K$.

Let $a_{1}, a_{2} \in A(x, y)$ and $\lambda \in[0,1]$. Put $a=\lambda a_{1}+(1-\lambda) a_{2}$. Since $a_{1}, a_{2} \in S_{1}(x)$ and $S_{1}(x)$ is convex set, we have $a \in S_{1}(x)$. By (ii), $F_{1}(\cdot, y, z)$ is properly $C$-quasiconvex. Without loss of generality, we can assume that

$$
F_{1}\left(a_{1}, y, z\right) \subset F_{1}\left(\lambda a_{1}+(1-\lambda) a_{2}, y, z\right)+C
$$

We claim that $a \in A(x, y)$. In fact, if $a \notin A(x, y)$, then there exists $z^{*} \in S_{1}(x)$ such that

$$
F_{1}\left(a, y, z^{*}\right) \nsubseteq C
$$

It follows that

$$
F_{1}\left(a_{1}, y, z^{*}\right) \subset F_{1}\left(\lambda a_{1}+(1-\lambda) a_{2}, y, z^{*}\right)+C \nsubseteq C+C \subset C,
$$

which contradicts to $a_{1} \in A(x, y)$. Therefore $a \in A(x, y)$ and hence $A(x, y)$ is a convex subset of $K$. Similarly, we have $B(x, y)$ is convex subset of $K$.

Step 3. Show that $A(x, y)$ and $B(x, y)$ are closed subsets of $K$.

Let $\left\{a_{\alpha}\right\}$ be a sequence in $A(x, y)$ such that $a_{\alpha} \rightarrow a^{*}$. Thus, we have $a_{\alpha} \in S_{1}(x)$. Since $S_{1}(x)$ is a closed subset of $K$, it follows that $a^{*} \in S_{1}(x)$. By the lower semicontinuity of $S_{1}$ and Lemma 2.5(iii), for any $z^{*} \in S_{1}(x)$ and any net $\left\{x_{\alpha}\right\} \rightarrow x$, there exists a net $\left\{z_{\alpha}\right\}$ such that $z_{\alpha} \in S_{1}\left(x_{\alpha}\right)$ and $z_{\alpha} \rightarrow z^{*}$. This implies that

$$
F_{1}\left(a_{\alpha}, y, z_{\alpha}\right) \subset C
$$

Since $F_{1}(\cdot, y, \cdot)$ is lower $(-C)$-continuous, for any neighbourhood $U$ of the origin in $Z$, there is a subnet $\left\{a_{\beta}, z_{\beta}\right\}$ of $\left\{a_{\alpha}, z_{\alpha}\right\}$ such that

$$
F_{1}\left(a^{*}, y, z^{*}\right) \subset F_{1}\left(a_{\beta}, y, z_{\beta}\right)+U+C
$$

From (3.5) and (3.6), we have

$$
F_{1}\left(a^{*}, y, z^{*}\right) \subset U+C
$$


We claim that $F_{1}\left(a^{*}, y, z^{*}\right) \subset C$. Assume that there exists $p \in F_{1}\left(a^{*}, y, z^{*}\right)$ and $p \notin C$. Thus, we note that $0 \notin(C-p)$ and $C-p$ is closed. Hence $Z \backslash(C-p)$ is open and $0 \in Z \backslash(C-p)$. Since $Z$ is a locally convex space, there exists a neighbourhood $U_{0}$ of the origin such that $U_{0} \subset Z \backslash(C-p)$ is convex and $U_{0}=-U_{0}$. This implies that $0 \notin U_{0}+(C-p)$, that is, $p \notin U_{0}+C$, which is a contradiction. Therefore $F_{1}\left(a^{*}, y, z^{*}\right) \subset C$. This mean that $a^{*} \in A(x, y)$ and so $A(x, y)$ is a closed subset of $K$. Similarly, we have $B(x, y)$ is a closed subset of $K$.

Step 4. Show that $A(x, y)$ and $B(x, y)$ are upper semicontinuous.

Let $\left\{\left(x_{\alpha}, y_{\alpha}\right): \alpha \in I\right\} \subset K \times D$ be given such that $\left(x_{\alpha}, y_{\alpha}\right) \rightarrow(x, y) \in K \times D$, and let $a_{\alpha} \in A\left(x_{\alpha}, y_{\alpha}\right)$ such that $a_{\alpha} \rightarrow a$. Since $a_{\alpha} \in S_{1}\left(x_{\alpha}\right)$ and $S_{1}$ is upper semicontinuous, it follows by Lemma 2.5(ii) that $a \in S_{1}(x)$. We now claim that $a \in A(x, y)$. Assume that $a \notin A(x, y)$. Then, there exists $z^{*} \in S_{1}(x)$ such that

$$
F_{1}\left(a, y, z^{*}\right) \nsubseteq C,
$$

which implies that there is a neighbourhood $U_{0}$ of the origin in $Z$ such that

$$
F_{1}\left(a, y, z^{*}\right)+U_{0} \nsubseteq C
$$

Since $F_{1}$ is upper $C$-continuous, for any neighbourhood $U$ of the origin in $Z$, there exists a neighbourhood $U_{1}$ of $\left(a, y, z^{*}\right)$ such that

$$
F_{1}(\widehat{a}, \widehat{y}, \widehat{z}) \subset F_{1}\left(a, y, z^{*}\right)+U+C, \quad \forall(\widehat{a}, \widehat{y}, \widehat{z}) \in U_{1}
$$

Without loss of generality, we can assume that $U_{0}=U$. This implies that

$$
F_{1}(\widehat{a}, \widehat{y}, \widehat{z}) \subset F_{1}\left(a, y, z^{*}\right)+U_{0}+C \nsubseteq C+C \subset C, \quad \forall(\widehat{a}, \widehat{y}, \widehat{z}) \in U_{1}
$$

Thus there is $\alpha_{0} \in I$ such that

$$
F_{1}\left(a_{\alpha}, y_{\alpha}, z_{\alpha}\right) \nsubseteq C, \quad \forall \alpha \geq \alpha_{0}
$$

which contradicts to $a_{\alpha} \in A\left(x_{\alpha}, y_{\alpha}\right)$. Hence $a \in A(x, y)$ and, therefore, $A$ is a closed mapping. Since $K$ is a compact set and $A(x, y)$ is a closed subset of $K$, we note that $A(x, y)$ is compact. Then, $\overline{A(x, y)}$ is also compact. Hence, by Lemma 2.5(i), $A(x, y)$ is an upper semicontinuous mapping. Similarly, we note that $B(x, y)$ is an upper semicontinuous mapping.

Step 5. Show that SGSVQEP has a solution.

Define the set-valued mapping $H_{a}: K \times D \rightarrow 2^{K \times D}$ and $G_{b}: K \times D \rightarrow 2^{K \times D}$ by

$$
\begin{aligned}
& H_{a}(x, y)=\left(A(x, y), T_{1}(a)\right) \quad \forall(x, y) \in K \times D, \\
& G_{b}(x, y)=\left(B(x, y), T_{2}(b)\right) \quad \forall(x, y) \in K \times D .
\end{aligned}
$$

Then, $H_{a}$ and $G_{b}$ are upper semicontinuous and, for all $(x, y) \in K \times D, H_{a}(x, y)$, and $G_{b}(x, y)$ are nonempty closed convex subsets of $K \times D$. 
Define the set-valued mapping $M:(K \times D) \times(K \times D) \rightarrow 2^{(K \times D) \times(K \times D)}$ by

$$
M((x, y),(u, v))=\left(H_{u}(x, y), G_{x}(u, v)\right), \quad \forall((x, y),(u, v)) \in(K \times D) \times(K \times D) .
$$

Then, $M$ is also upper semicontinuous and, for all $((x, y),(u, v)) \in(K \times D) \times(K \times D)$, $M((x, y),(u, v))$ is a nonempty closed convex subset of $(K \times D) \times(K \times D)$. By Lemma 2.4, there exists a point $((\bar{x}, \bar{y}),(\bar{u}, \bar{v})) \in(K \times D) \times(K \times D)$ such that $((\bar{x}, \bar{y}),(\bar{u}, \bar{v})) \in M((\bar{x}, \bar{y}),(\bar{u}, \bar{v}))$, that is

$$
(\bar{x}, \bar{y}) \in H_{\bar{u}}(\bar{x}, \bar{y}), \quad(\bar{u}, \bar{v}) \in G_{\bar{x}}(\bar{u}, \bar{v})
$$

This implies that $\bar{x} \in A(\bar{x}, \bar{y}), \bar{y} \in T_{1}(\bar{u}), \bar{u} \in B(\bar{u}, \bar{v})$, and $\bar{v} \in T_{2}(\bar{x})$. Then, there exists $(\bar{x}, \bar{u}) \in K \times K$ and $\bar{y} \in T_{1}(\bar{u}), \bar{v} \in T_{2}(\bar{x})$ such that $\bar{x} \in S_{1}(\bar{x}), \bar{u} \in S_{2}(\bar{u})$,

$$
F_{1}(\bar{x}, \bar{y}, z) \subset C, \quad \forall z \in S_{1}(\bar{x}), \quad F_{2}(\bar{u}, \bar{v}, z) \subset C, \quad \forall z \in S_{2}(\bar{u})
$$

Hence SGSVQEP has a solution.

Step 6. Show that the set of solutions of SGSVQEP is closed.

Let $\left\{\left(x_{\alpha}, u_{\alpha}\right): \alpha \in I\right\}$ be a net in the set of solutions of SGSVQEP such that $\left(x_{\alpha}, u_{\alpha}\right) \rightarrow$ $\left(x^{*}, u^{*}\right)$. By definition of the set of solutions of SGSVQEP, we note that there exist $v_{\alpha} \in T_{1}\left(x_{\alpha}\right)$, $y_{\alpha} \in T_{2}\left(u_{\alpha}\right), x_{\alpha} \in S_{1}\left(x_{\alpha}\right)$, and $u_{\alpha} \in S_{2}\left(u_{\alpha}\right)$ satisfying

$$
F_{1}\left(x_{\alpha}, y_{\alpha}, z\right) \subset C, \quad \forall z \in S_{1}\left(x_{\alpha}\right), \quad F_{2}\left(u_{\alpha}, v_{\alpha}, z\right) \subset C, \quad \forall z \in S_{2}\left(u_{\alpha}\right)
$$

Since $S_{1}$ and $S_{2}$ are continuous closed valued mappings, we obtain $x^{*} \in S_{1}\left(x^{*}\right)$ and $u^{*} \in$ $S_{2}\left(u^{*}\right)$. Let $v_{\alpha} \rightarrow v^{*}$ and $y_{\alpha} \rightarrow y^{*}$. Since $T_{1}$ and $T_{2}$ are upper semicontinuous closed valued mappings, it follows by Lemma 2.5(ii) that $T_{1}$ and $T_{2}$ are closed. Thus, we note that $v^{*} \in T_{1}\left(x^{*}\right)$ and $y^{*} \in T_{2}\left(u^{*}\right)$. Since $F_{1}\left(\cdot, y^{*}, \cdot\right)$ and $F_{2}\left(\cdot, v^{*}, \cdot\right)$ are lower $(-C)$-continuous, we have

$$
F_{1}\left(x^{*}, y^{*}, z\right) \subset C, \quad \forall z \in S_{1}\left(x^{*}\right), \quad F_{2}\left(u^{*}, v^{*}, z\right) \subset C, \quad \forall z \in S_{2}\left(u^{*}\right)
$$

This means that $\left(x^{*}, u^{*}\right)$ belongs to the set of solutions of SGSVQEP. Hence the set of solutions of SGSVQEP is closed set. This completes the proof.

If we take $S=S_{1}=S_{2}, F=F_{1}=F_{2}$, and $T=T_{1}=T_{2}$. Then, from Theorem 3.1, we derive the following result.

Corollary 3.2. Let $S: K \rightarrow 2^{K}$ be a continuous set-valued mapping such that for any $x \in K, S(x)$ is nonempty closed convex subset of $K$. Let $T: K \rightarrow 2^{D}$ be an upper semicontinuous set-valued mapping such that for any $x \in K, T(x)$ is a nonempty closed convex subset of $D$ and $F: K \times D \times K \rightarrow$ $2^{Z}$ be set-valued mapping satisfy the following conditions:

(i) for all $(x, y) \in K \times D, F(x, y, S(x)) \subset C$,

(ii) for all $(y, z) \in D \times K, F(\cdot, y, z)$ is properly $C$-quasiconvex, 
(iii) $F(\cdot, \cdot, \cdot)$ is an upper $C$-continuous,

(iv) for all $y \in D, F(\cdot, y, \cdot)$ is a lower $(-C)$-continuous,

(v) if $x \in S(x)$ and $u \in S(u)$ then $T(x)=T(u)$.

Then, GSVQEP has a solution. Moreover, the set of all solution of GSVQEP is closed.

Now we give an example to explain that Theorem 3.1 is applicable.

Example 3.3. Let $X=Y=Z=\mathbf{R}, C=[0,+\infty)$, and $K=D=[0,1]$. For each $x \in K$, let $S_{1}(x)=[x, 1], S_{2}(x)=[0, x]$ and $T_{1}(x)=[1-x, 1], T_{2}(x)=[x, 1]$. We consider the set-valued mappings $F_{1}, F_{2}: K \times D \times K \rightarrow 2^{Z}$ defined by

$$
\begin{array}{ll}
F_{1}(x, y, z)=[x-y+z,+\infty) & \forall(x, y, z) \in K \times D \times K \\
F_{2}(x, y, z)=[y-x+z,+\infty) & \forall(x, y, z) \in K \times D \times K .
\end{array}
$$

Then, it is easy to check that all of condition (i)-(iv) in Theorem 3.1 are satisfied. Hence, by Theorem 3.1, SGSVQEP has a solution. Let $E$ be the set of all strong solutions for SGSVQEP. Then, we note that

$$
\begin{aligned}
E= & \left\{(\bar{x}, \bar{u}, \bar{y}, \bar{v}) \in K \times K \times T_{2}(\bar{u}) \times T_{1}(\bar{x}): \bar{x} \in S_{1}(\bar{x}), \bar{u} \in S_{2}(\bar{u})\right. \text { such that } \\
& \left.F_{1}(\bar{x}, \bar{y}, z) \subset C, \forall z \in S_{1}(\bar{x}), F_{2}(\bar{u}, \bar{v}, z) \subset C, \forall z \in S_{2}(\bar{u})\right\} \\
= & \bigcup_{1 / 3 \leq a \leq 0.5}(\{a\} \times[1-a, 2 a] \times[0,1-a] \times[1-a, 1]) .
\end{aligned}
$$

\section{Acknowledgments}

The authors would like to thank the referees for the insightful comments and suggestions. S. Plubtieng the Thailand Research Fund for financial support under Grants no. BRG5280016. Moreover, K. Sitthithakerngkiet would like to thanks the Office of the Higher Education Commission, Thailand for supporting by grant fund under Grant no. CHE-Ph.D-SWRG/41/2550, Thailand.

\section{References}

[1] M. Bianchi and S. Schaible, "Generalized monotone bifunctions and equilibrium problems," Journal of Optimization Theory and Applications, vol. 90, no. 1, pp. 31-43, 1996.

[2] E. Blum and W. Oettli, "From optimization and variational inequalities to equilibrium problems," The Mathematics Student, vol. 63, no. 1-4, pp. 123-145, 1994.

[3] W. Oettli and D. Schläger, "Existence of equilibria for monotone multivalued mappings," Mathematical Methods of Operations Research, vol. 48, no. 2, pp. 219-228, 1998.

[4] Q. H. Ansari, W. Oettli, and D. Schläger, "A generalization of vectorial equilibria," Mathematical Methods of Operations Research, vol. 46, no. 2, pp. 147-152, 1997.

[5] Q. H. Ansari, S. Schaible, and J. C. Yao, "System of vector equilibrium problems and its applications," Journal of Optimization Theory and Applications, vol. 107, no. 3, pp. 547-557, 2000.

[6] M. Bianchi, N. Hadjisavvas, and S. Schaible, "Vector equilibrium problems with generalized monotone bifunctions," Journal of Optimization Theory and Applications, vol. 92, no. 3, pp. 527-542, 1997. 
[7] G. Debreu, "A social equilibrium existence theorem," Proceedings of the National Academy of Sciences of the United States of America, vol. 38, pp. 886-893, 1952.

[8] J.-Y. Fu, "Generalized vector quasiequilibrium problems," Mathematical Methods of Operations Research, vol. 52, no. 1, pp. 57-64, 2000.

[9] F. Giannessi, Vector Variational Inequilities and Vector Equilibria, Mathematical Theories, vol. 38, Kluwer Academic Publishers, Dordrecht, The Netherlands, 2000.

[10] X. Gong, "Strong vector equilibrium problems," Journal of Global Optimization, vol. 36, no. 3, pp. 339 $349,2006$.

[11] X. H. Gong, "Efficiency and Henig efficiency for vector equilibrium problems," Journal of Optimization Theory and Applications, vol. 108, no. 1, pp. 139-154, 2001.

[12] R. B. Holmes, Geometric Functional Analysis and Its Application, Graduate Texts in Mathematics, no. 2, Springer, New York, NY, USA, 1975.

[13] S. H. Hou, X. H. Gong, and X. M. Yang, "Existence and stability of solutions for generalized Ky Fan inequality problems with trifunctions," Journal of Optimization Theory and Applications, vol. 146, no. 2, pp. 387-398, 2010.

[14] N. J. Huang, J. Li, and H. B. Thompson, "Stability for parametric implicit vector equilibrium problems," Mathematical and Computer Modelling, vol. 43, no. 11-12, pp. 1267-1274, 2006.

[15] S. J. Li, K. L. Teo, and X. Q. Yang, "Generalized vector quasiequilibrium problems," Mathematical Methods of Operations Research, vol. 61, no. 3, pp. 385-397, 2005.

[16] X. Long, N. Huang, and K. Teo, "Existence and stability of solutions for generalized strong vector quasiequilibrium problem," Mathematical and Computer Modelling, vol. 47, no. 3-4, pp. 445-451, 2008.

[17] Q. H. Ansari, W. Oettli, and D. Schiager, "A generalization of vectorial equilibria," in Proceedings of the 2nd International Symposium on Operations Research and Its Applications (ISORA '96), vol. 1114, pp. 181-185, World Publishing, Guilin, China, December1996.

[18] Q. H. Ansari, W. K. Chan, and X. Q. Yang, "The system of vector quasiequilibrium problems with applications," Journal of Global Optimization, vol. 29, no. 1, pp. 45-57, 2004.

[19] Q. H. Ansari, "Existence of solutions of systems of generalized implicit vector quasiequilibrium problems," Journal of Mathematical Analysis and Applications, vol. 341, no. 2, pp. 1271-1283, 2008.

[20] Q. H. Ansari and Z. Khan, "System of generalized vector quasiequilibrium problems with applications," in Mathematical Analysis and Applications, S. Nanda and G. P. Rajasekhar, Eds., vol. 7, pp. 1-13, Narosa, New Delhi, India, 2004.

[21] Q. H. Ansari, L. J. Lin, and L. B. Su, "Systems of simultaneous generalized vector quasiequilibrium problems and their applications," Journal of Optimization Theory and Applications, vol. 127, no. 1, pp. 27-44, 2005.

[22] Q. H. Ansari, S. Schaible, and J.-C. Yao, "The system of generalized vector equilibrium problems with applications," Journal of Global Optimization, vol. 22, no. 1-4, pp. 3-16, 2002.

[23] L.-J. Lin, "System of generalized vector quasiequilibrium problems with applications to fixed point theorems for a family of nonexpansive multivalued mappings," Journal of Global Optimization, vol. 34, no. 1, pp. 15-32, 2006.

[24] J.-P. Aubin and I. Ekeland, Applied Nonlinear Analysis, Pure and Applied Mathematics, John Wiley \& Sons, New York, NY, USA, 1984. 